\title{
Interdisciplinaridade no ensino prático em Residência Multiprofissional em Saúde
}

\author{
Interdisciplinarity in the practical teaching in Multiprofessional Residence in Health
}

Interdisciplinaridade en la enseñanza práctica en Residencia Multiprofesional en Salud

Gleyce Pinto Girard ${ }^{1 \star}$, Daniele Melo Sardinha², Marcia Helena Machado Nascimento ${ }^{1}$, Renato da Costa Teixeira ${ }^{1}$, Sâmia Cristine Rabelo Borges ${ }^{1}$.

\section{RESUMO}

Objetivo: Analisar as práticas de preceptores da Residência Multiprofissional em Saúde de um hospital escola de Belém sobre interdisciplinaridade. Método: Estudo descritivo com abordagem qualitativa e auxílio do software Alceste 2015, realizada no período de março a junho de 2017. Os dados foram coletados em um hospital escola em Belém do Pará com preceptores de Residência Multiprofissional em Saúde em grupo focal, norteado por um roteiro semiestruturado com perguntas abertas. Resultados: A participação de 14 preceptores de seis diferentes categorias profissionais evidenciou conhecimento superficial sobre interdisciplinaridade, as práticas de ensino com os residentes são desarticuladas, os preceptores não recebem educação permanente sobre este tema. Conclusão: Aponta-se para a necessidade de formação profissional a preceptores sobre o tema interdisciplinaridade para o fortalecimento da integralidade no Sistema Único de Saúde.

Descritores: Pesquisa Interdisciplinar, Preceptoria, Formação Profissional.

\begin{abstract}
Objective: To analyze the practices of preceptors of the Multiprofessional Health Residency of a school hospital in Belém on interdisciplinarity. Method: A descriptive study with a qualitative approach and help from the Alceste 2015 software, carried out from March to June 2017. Data were collected at a school hospital in Belém do Pará with preceptors of Multiprofessional Health Residency in a focal group, guided by a semistructured script with open questions. Results: The participation of 14 preceptors from six different professional categories showed superficial knowledge about interdisciplinarity, teaching practices with the residents are disarticulated, the preceptors do not receive permanent education on this subject. Conclusion: It is pointed out to the need for professional training to preceptors on the interdisciplinarity theme for the strengthening of integrality in the Unified Health System.
\end{abstract}

Descriptors: Interdisciplinary Research, Preceptorship, Vocational Training.

\section{RESUMEN}

Objetivo: Analizar las prácticas de preceptores de la Residencia Multiprofesional en Salud de un hospital escuela de Belém sobre interdisciplinaridad. Método: Estudio descriptivo con abordaje cualitativo y auxilio del software Alceste 2015, realizado en el período de marzo a junio de 2017. Los datos fueron recolectados en un hospital escolar en Belém do Pará con preceptores de Residencia Multiprofesional en Salud en grupo focal, orientado por uno el itinerario semiestructurado con preguntas abiertas. Resultados: La participación de 14

\footnotetext{
1 Universidade do Estado do Pará - UEPA, Belém-PA. * E-mail: gleycegirard@hotmail.com

2 Centro Universitário Metropolitano da Amazônia - UNIFAMAZ, Belém-PA.
} 
preceptores de seis diferentes categorías profesionales evidenció conocimiento superficial sobre interdisciplinariedad, las prácticas de enseñanza con los residentes son desarticuladas, los preceptores no reciben educación permanente sobre este tema. Conclusión: Se apunta a la necesidad de formación profesional a preceptores sobre el tema interdisciplinariedad para el fortalecimiento de la integralidad en el Sistema Único de Salud.

Descriptores: Investigación Interdisciplinaria, Preceptoria, Formación Profesional.

\section{INTRODUÇÃO}

A Residência Multiprofissional em Saúde (RMS), tem origem em 30 de junho de 2005, pela Lei no 11.129, que instituiu o Programa Nacional de Inclusão de Jovens no mercado de trabalho, norteada pelos princípios e diretrizes do Sistema Único de Saúde (SUS), atendendo necessidades loco-regionais, envolvendo 13 profissões da saúde (BRASIL MDE, 2007). Regida por uma Comissão Nacional de Residência Multiprofissional em Saúde (CNRMS) que regula, avalia e supervisiona as (RMS) em todo território nacional. (BRASIL MDS, 2012).

Esta pós graduação tem como objetivo articulação entre academia e serviço para a formação de profissionais com uma concepção interdisciplinar para atuar no SUS, pela lógica do trabalho em equipe multiprofissional, para promoção de uma assistência integral ao cidadão (LOBATO CP, 2010). Neste sentido, preceptores de diversas formações da área da saúde, vinculados ao SUS, realizam prática de ensino em RMS, que, devem dispor do uso da interdisciplinaridade para ressignificar o processo de ensinoaprendizagem, de modo a garantir o exercício da integralidade por meio de troca de conhecimentos, provocando a ruptura de valores tradicionais na saúde como a compartimentalização do saber e a departamentalização profissional, para superar as fronteiras disciplinares, enaltecer a importância do trabalho em equipe e, assim, resolver complexidades na saúde (BORGES MJL, et al., 2012).

Para Silva VCD et al. (2014), o preceptor tem um papel fundamental na reorientação de profissionais de saúde, sendo possível inferir que este é um agente de prática social, mediador, supervisor, orientador, no processo de formação em serviço de saúde para a construção de conhecimento e resolução de problemas de saúde numa concepção de assistência integral.

A interdisciplinaridade tem sido considerada uma metodologia proximal do princípio da integralidade, denotando interação, reciprocidade, diálogo e consequentemente transformação, além de efetuar resolução de problemas de maneira articulada (FAZENDA ICA, et al., 2013).

O'Reilly P et al. (2017), refere que as bases de evidências são limitadas no âmbito da interdisciplinaridade entre profissionais de saúde, pois a experiência profissional dos mesmos não reflete a atuação interdisciplinar, isto se deve à falta de política direcionada a implementação de um modelo de atuação em equipe. Evidências produzidas no Canadá, Suécia, Reino Unido e Austrália chamam atenção para investimentos e incentivos direcionados a melhoria da comunicação entre profissionais de saúde com reuniões regulares que oportunize a tomada de decisão em equipe, pois se repercute com sucesso na melhoria do atendimento, das funções, metas e visão compartilhada.

Para Assein J et al. (2016) a base do cuidado interdisciplinar é não somente o acordo de ideias entre membros de uma equipe, mas as relações de comunicação e negociação clínica contínua que delibera estratégias para o cuidado e controle de doenças.

Nesse sentido, compreendendo que o universo da profissão se completa, na teoria e na legislação, nesta pesquisa, de forma norteadora, utiliza-se, a Lei no 11.129/06/2005, que instituiu o Programa Nacional de Inclusão de Jovens que norteou a Portaria Interministerial no 2.538, e instituiu o Grupo de Trabalho da Residência Multiprofissional em Saúde (BRASIL MDE, 2015), além de artigos, livros, preferencialmente, lançados nos últimos 15 anos, dada a escassez de material bibliográfico, sobre o tema, a disposição, publicado nos últimos 05 anos. 
Sobre esse fator limitante, é importante enunciar que, apesar de uma quantidade considerável de artigos, que versam sobre o tema proposto, terem sido editados, assim como ocorre com este trabalho de pesquisa, todos os autores recorreram a fontes anteriores aos últimos cinco anos, com raras exceções, ou fizeram uso do recurso do apud, seja pela necessidade de se fazer a citação indireta ou para dar a impressão de que suas fontes cumprem a esse quesito cronológico.

Levando-se em consideração o fato de que cada especialidade é um universo isolado do todo e que a atenção em saúde prioriza ações que possibilitem responder às necessidades sociais de saúde, potencializando formas amplas de intervenção em diversos aspectos que determinam o processo saúdedoença (GUEDES LE e JUNIOR MF (2010), entende-se, que é importante refletir sobre propostas de formação profissional que integrem o ensino-serviço, o saber e as práticas de diferentes especialistas, gestores e clientes a fim de encontrar soluções compartilhadas para os problemas de saúde do cotidiano, colocando-se em prática a integralidade de atenção à saúde. O presente trabalho de pesquisa tem como objetivo, analisar os saberes e as práticas de preceptores sobre interdisciplinaridade em uma determinada Residência Multiprofissional.

\section{MÉTODOS}

Estudo descritivo com abordagem qualitativa. A técnica de coleta de dados envolveu entrevista em Grupo Focal, norteado por um roteiro de entrevista semiestruturada com perguntas abertas. No período de março a junho de 2017. Os entrevistados foram preceptores da Residência Multiprofissional em Saúde de uma Fundação Hospital de Ensino em Belém do Pará. Houve a submissão e parecer substanciado do CEP de número 1855190. Após a coleta dos dados, foi realizada a análise de conteúdo, conforme orienta Bardin $\mathrm{L}$ (2016). Para auxiliar a análise de conteúdo fez-se uso do software Alceste 2015 (Analyse Lexicale par Contexte d'un Ensenble de Segments de Texte) de análise de dados qualitativos.

Uma vez que os resultados foram gerados, realizou-se inferências e interpretações correlacionadas aos objetivos previstos. A confrontação sistemática do material e as inferências alcançada serviram de base à outras análises dispostas em torno de novas dimensões teóricas respondendo cientificamente às questões norteadoras.

\section{RESULTADOS}

Os participantes foram 14 preceptores com idade entre 35 a 56 anos: 3 enfermeiros, 2 psicólogos, 2 fisioterapeutas, 3 nutricionistas, 2 assistentes sociais e 2 terapeutas ocupacionais. Todos compõem a equipe Multiprofissional do hospital e concomitantemente exercem a função assistencial e de preceptoria na Residência Multiprofissional, o tempo de atividade profissional, destes, em serviço assistencial, neste hospital, varia entre dois anos a trinta e dois anos; e em preceptoria, varia entre dois anos a quatro anos.

O software Alceste gerou classes, a partir da análise léxica dos dados coletados, as classes foram nomeadas e agrupadas em 3 eixos, de acordo com a sua semelhança em relação ao assunto. São eles: Formação do preceptor; Comunicação Interdisciplinar.

\section{Eixo 1: Formação Do Preceptor}

A Residência Multiprofissional foi estabelecida no hospital em estudo no ano de 2012. Inicialmente, segundo os preceptores participantes, não foi possível abster-se da função de preceptoria, pois todos foram escolhidos para a função e não houve uma qualificação profissional prévia para desempenhar esta atividade profissional, por este motivo, sentiram muita dificuldade ao serem inseridos no campo da preceptoria, as falas abaixo confirmam isto: 
"A minha chefa me disse, tu vás ser preceptora. Eu disse assim: espera aí porque eu nem sei o que é ser preceptora". ${ }^{* \star * *}$ "entrevistado 13

A ausência de uma formação para atuação no ensino, deixa lacunas que são sentidas pelo preceptor na vivência da prática em preceptoria, são elas: pobre conhecimento sobre a função do preceptor, sobre a função do residente, sobre avaliar o aprendizado do residente, conduzir estudos de casos clínicos e ser qualificado para o ensino prático.

"Precisamos nos inteirar mais de pesquisas científicas, aprender a avaliar o residente. Não existe uma análise crítica, não temos critérios claros para avaliar, a gente fica com medo de dar uma nota baixa, a gente fica com medo de ser punitivo ou de ser permissivo". "*** *entrevistado 6

\section{Eixo 2: Comunicação Interdisciplinar}

Esta categoria desvela o saber e a prática do preceptor sobre interdisciplinaridade. O entendimento destes sobre interdisciplinaridade, se mostrou superficial elucidando noções gerais, evidente nas falas abaixo:

"É a soma de vários conhecimentos de áreas diferentes, a psicologia, a enfermagem, a médica, porém o objetivo é único, a gente pega o conhecimento específico de cada área e tenta fazer uma agregação, uma ajuda mútua". ${ }^{* * * \star}$ *entrevistado 1

"É um conjunto de ações integradas, o olhar holístico que é muito enfatizado na faculdade. Não sei se é coisa da formação profissional ou da personalidade. É algo da formação ou é algo da personalidade." **** *entrevistado 3

Os preceptores não relacionaram interdisciplinaridade à atividade pedagógica, à teoria e a prática. Todos focaram apenas na comunicação entre profissionais para expressarem suas ideias sobre o tema.

"No próprio hospital só existiam médico e enfermeira, outras categorias foram se agregando bem depois, aí veio a fisioterapeuta, terapeuta ocupacional, já é um trabalho maior, assistente social, psicólogo, que anteriormente não faziam parte da equipe, até um certo tempo, dentro dos hospitais. Como professor, fui há muito tempo, professor de prática em hospital, o que eu via no meu ponto de vista, nos víamos alunos da mesma instituição passavam um pelo outro e não se reconheciam, era de medicina, era de fisioterapia, que tinham aula na mesma faculdade, a residência possibilita mais isso, os residentes se reconhecem, eles fazem uma residência multiprofissional, mas como eles estão aqui, eles acabam se reconhecendo, pelas clínicas que eles passam, então acaba se consolidando a interdisciplinaridade." ${ }^{* * * *}$ "entrevistado 13

"Eu acho que é trabalhar não apenas vendo o seu trabalho, não apenas trabalhando no seu quadrado, mas vendo que o trabalho do outro também interfere no seu. É passear por essas categorias, entender que é uma situação dinâmica, que você não está fazendo nada sozinho e que você precisa integrar, ter conhecimento interdisciplinar, você precisa trabalhar junto, ajudar um ao outro, aí isso é interdisciplinaridade." **** *entrevistado 9

Quanto a formação acadêmica e profissional para atuar com interdisciplinaridade, todos os participantes referiram que não tiveram aproximação deste tema em capacitações, enfatizaram a falta de habilidade e competência, pois não foram preparados para o agir com interdisciplinaridade.

"Não tivemos treinamento sobre isso, mas acho que a gente traz a interação de berço, tem gente que tem uma certa dificuldade para interagir. Depende também muito da faculdade que você se forma." ${ }^{* * * *}$ entrevistado 9

"Nós profissionais não tivemos preparo para trabalhar interdisciplinaridade, até porque no próprio hospital só existiam médico e enfermeira, outras categorias foram se agregando bem depois, aí veio a fisioterapeuta, terapeuta ocupacional, já é um trabalho maior, assistente social, psicólogo, que 
anteriormente não faziam parte da equipe, até um certo tempo, dentro dos hospitais." **** *entrevistado 12

Sobre a prática interdisciplinar, os participantes referiram que decidem sobre as condutas assistenciais aos pacientes, por meio de conversas isoladas entre especialistas, de maneira que um repassa informações importantes ao outro sobre as necessidades clínicas dos pacientes, o mesmo ocorre entre os residentes.

"Quando os residentes têm alguma dúvida sobre a patologia do paciente, a gente vai, conversa com o médico, ele explica alguma coisa que às vezes a gente não está bem inteirado sobre a doença. Às vezes é com o nutricionista, a gente vai e conversa, tira uma dúvida. Os residentes, às vezes, têm alguma dúvida sobre fisioterapia, sobre alguns exercícios que o paciente faz e vai até $o$ fisioterapeuta perguntar." **** *entrevistado 11

"Tem situação de paciente que precisa, conforme o quadro clinico, de acompanhante, ele é depressivo, pode ter o risco de suicídio, aí o psicólogo detectou isso, aí ele passa a informação para a enfermagem que olha o que paciente precisa e chama o assistente social para contactar a família." **** *entrevistado 7

As citações dos participantes relacionam interdisciplinaridade com as diferentes personalidades de especialistas, no grupo, resistentes à comunicação, restringindo-se a um trabalho solitário, como expressado:

"Vai da condição humana, isso tem muito da personalidade, tem pessoas que tem facilidade de comunicação, tem pessoas que não tem facilidade, mesmo, não é questão profissional, é mesmo social, fora daqui tem dificuldade de conversar, de interagir com os outros. Então em cima disso existe essa preparação na academia, que pode ser intensificada. Tem as dificuldades individuais, porque cada residente é de personalidade diferente." ${ }^{* * * *}$ entrevistado 7

"A partir do momento em que se faz uma proposta do hospital escola e a classe médica é separada, então a gente não pode falar de interdisciplinaridade aqui. A equipe médica é muito isolada, eles não interagem de jeito nenhum. Eles estudam sozinhos os casos clínicos dos pacientes, eles nunca convidam as outras categorias para formar um cuidado integral." **** *entrevistado 4

\section{DISCUSSÃO}

A figura do preceptor, em Programas de Residência Multiprofissional tem sido vista como a de um profissional experiente em prática clínica e com vasto conhecimento técnico associado há anos de exercício clínico. Embora, o tempo de competência de prática clínica seja significativo para a definição de um bom profissional técnico, não é necessariamente suficiente para definir a qualidade de um professor ou um bom preceptor (IZECKSOHN MMV, et al., 2017).

Bispo EPEF (2013), realizaram entrevistas com nove preceptores atuantes de uma Estratégia de Saúde da Família em Maceió, dentre estes, apenas um profissional obteve capacitação para preceptoria.

Silva ABDA ,et al., (2010) em um estudo realizado em Hospitais Universitários do Rio de Janeiro, UNIRIO, evidenciou que um grande percentual de preceptores desconhecia suas atribuições. O mesmo estudo fez 0 levantamento das necessidades dos preceptores, para elaborar estratégias de capacitação destes, por meio de Educação Permanente e orientá-los sobre os processos educacionais na formação para o SUS.

No que tange ao conceito de preceptor, Autonomo FRDOM, et al., (2015), fizeram um estudo bibliográfico e analisaram os conceitos, atividades e características da preceptoria nas publicações brasileiras em saúde, no período de 2002 a 2012, obtiveram termos relevantes para concebe-los: "docente-clínico", "educador", "docente", "tutor", "profissional de saúde", "membro da equipe de supervisão", "o que oferece apoio 
pedagógico", "o que oferece suporte técnico assistencial" ou ainda, contraditoriamente, como "o profissional da academia, que se desloca para o território em que o residente é membro da equipe".

O preceptor é um docente-clínico com bagagem teórica e clínica, tem a função de supervisionar a atividade prática do estudante, orientar, educar, trocar, construir e reconstruir conhecimentos, diagnosticar a compreensão do residente, ensinar realizando procedimentos técnicos, moderar na discussão de casos clínicos e desempenhar papel ético (BOTTI SHDO, 2012).

Conforme a resolução da Comissão Nacional de Residência Multiprofissional em Saúde - CNRMS № 2 de 13.04.2012, artigo 14, o papel do preceptor é exercer a função de orientador de referência para o(s) residente(s), no desempenho das atividades práticas, vivenciadas no cotidiano da atenção, gestão em saúde, facilitar a integração do(s) residente(s) com a equipe de saúde, usuários (indivíduos, família e grupos), residentes de outros programas, bem como com estudantes dos diferentes níveis de formação profissional, na saúde, que atuam no campo de prática (BRASIL MDS, 2012).

A interdisciplinaridade faz parte do exercício profissional do preceptor. Reis MBDF (2009), define que interdisciplinaridade no ensino é exercitar o resgate do pensamento de unidade do conhecimento humano que foi se especializando, com a expansão do conhecimento científico, de forma que as partes parecem estar separadas do todo, havendo a ruptura entre o saber e da realidade.

A ausência de qualificação de preceptores faz com que os estudantes passem pela prática assistencial, desempenhando um papel de observador de procedimentos desempenhados pelo preceptor, isto obscurece a percepção do educador e do educando, no processo ensino-aprendizagem, resumindo-se em uma prática social e não em uma prática problematizadora (SILVA SVD,et al., 2014).

Uma postura interdisciplinar à educação disciplinar é algo que incorpora as disciplinas, dando legitimidade e sentido às disciplinas, na vida das pessoas, de forma que necessita de tempo para discussão entre professores e estudantes, para haver interdisciplinaridade (FAZENDA ICA, 2014).

Borges MJL et al (2012), entendem que um grupo de profissionais com especialidades diferentes, que trabalha junto, é considerado como uma equipe multiprofissional, porém, para que o trabalho deste grupo possa se configurar em um atendimento interdisciplinar, deve haver diálogo entre seus membros, com objetivos definidos de estabelecer um projeto de intervenção integral e específico às necessidades de cada paciente.

O exercício interdisciplinar, em uma residência multiprofissional, pode ser desempenhado por equipes multiprofissionais, com reuniões planejadas semanalmente, com 5 horas de duração, com programação de atividades definidas que envolvam discussão individual e coletiva de todos os casos atendidos havendo 0 parecer de cada especialista envolvido, proporcionando aos residentes uma experiência de trabalho interdisciplinar. A postura interdisciplinar proporciona multiplicação do conhecimento, na área assistencial em saúde, melhor conhecimento sobre 0 trabalho de cada especialidade, envolvimento dos residentes e percepção ainda mais ampliada sobre o processo saúde-doença (REIS BAO e FARO A, 2016).

Uma atitude interdisciplinar promove abertura de conhecimento, concedida por respeito, paciência, desapego, coerência, ousadia, numa atitude de troca intersubjetiva, de parceria, aproximação, abertura para aquisição de saberes, de mudanças e renovação das práticas, trata-se da articulação entre a complexidade da construção dos saberes dos seres humanos, no tempo e no espaço (FAZENDA ICA, 2008).

Embora essa aproximação e interação com o diferente, interagir com o diferente cotidiano do outro pode causar estranheza, medo, insegurança e incertezas (BRANDÃO V, 2015).

Entretanto, a interdisciplinaridade tem sido vista como um caminho para ampliar conhecimento sobre determinado assunto, considerando conceitos sociais, integração cultural, forças sociais, conceitos disciplinares como psicologia, economia, antropologia, levantando novas questões no âmbito de paradigmas de determinados conceitos, sobre determinado assunto (MITTELMARK MB, et al., 2016). 
Nas ciências da saúde voltadas para a consolidação do SUS, a interdisciplinaridade tem uma correlação estreita com o princípio da integralidade, pois ambos possibilitam uma percepção holística do sujeito, relacionando o seu estado de saúde ao seu contexto histórico, social, político, familiar e ambiental. Entendese que a atenção integral é individual e coletiva simultaneamente, sendo impossível, portanto, o exercício profissional em saúde dissociar o homem de todos os aspectos que o circundam, sendo imprescindível, a necessidade de articulação entre integrantes da equipe multiprofissional para uma assistência integral ao indivíduo (HARTZ ZMDA e CONTADRIOPOULOS AP, 2004).

Fazenda ICA (2008), pontua alguns princípios para o agir com interdisciplinaridade, que são: o desapego da zona de conforto dos conhecimentos disciplinares de base; a humildade em compartilhar conhecimentos em revisar, refletir, analisar, contextualizar seu próprio conhecimento em relação ao do outro e até mesmo, (re) construir conhecimentos; ousadia em criar inovações com engajamento e comprometimento; o respeito consigo e ao trabalho do outro; a coerência ao construir conhecimentos e o saber esperar o tempo que cada um leva para assimilar e construir conhecimentos.

Uma abordagem educativa em saúde deve ter como prerrogativas principais, desenvolver momentos de construção de saber coletivo, democratização do saber, troca de experiências da realidade, agir e refletir na sua ação, cujo objetivo é desenvolver no aprendiz uma personalidade pensante, de raciocínio, considerando seus valores, construindo hábitos de responsabilidade, cooperação em grupo integrando o profissional de saúde no processo educativo bem como a comunidade que recebe a prestação de serviço (VASCONCELOS M, et al., 2009).

O agir com interdisciplinaridade no ensino de residência multiprofissional requer reflexão, observação, interação entre as especialidades profissionais, construção de espaços para a troca de conhecimentos teóricos e práticos contextualizados com realidade do SUS, assim é que enfatiza (BRANDÃO VMAT, 2009).

\section{CONCLUSÃO}

O estudo permitiu a análise do saber e das práticas de preceptores do PRMS de um hospital escola de Belém do Pará sobre interdisciplinaridade. Constatou-se que os preceptores apresentam conhecimento superficial sobre interdisciplinaridade, não realizam prática interdisciplinar com os residentes e não receberam educação permanente sobre este tema. Os mesmos reconhecem suas limitações profissionais para atuação interdisciplinar e também para atuação pedagógica em preceptoria, pois não tiveram formação educativa para exercer tal atividade.

O exercício interdisciplinar, na residência multiprofissional, deve ser desempenhado por equipes multiprofissionais, com planejamento e reuniões regulares com uma programação de atividades que envolva discussão individual e coletiva de todos os casos atendidos na clínica para proporcionar aos residentes uma experiência de trabalho interdisciplinar. As reuniões proporcionam multiplicação do conhecimento, na área assistencial em saúde, melhor conhecimento sobre o trabalho de cada especialidade, envolvimento dos residentes e percepção ainda mais ampliada sobre o processo saúde-doença.

\section{REFERÊNCIAS}

1. ASSELIN $J$ et al. Challenges in interdisciplinary weight management in primary care: lessons learned from the 5As Team study. Clinical Obesity, 2016 v. 6, n. 2, p. 124-132.

2. AUTONOMO FRDOM et al. A Preceptoria na Formação Médica e Multiprofissional com Ênfase na Atenção Primária-Análise das Publicações. Revista Brasileira De Educação Médica, 2015 v. 39, n. 2, p. 316-327.

3. BARDIN L. Análise de Contéudo. 2016.

4. BISPO EPEF. Interdisciplinaridade no Ensino em Saúde: O Olhas do Precptor na Estratégia de Saúde da Família. Dissertação (Mestrado em ensino na Saúde) UFA, 2013; 44 p.

5. BORGES MJL, SAMPAIO AS, GURGEL IGD. Trabalho em equipe e interdisciplinaridade: desafios para a efetivação da integralidade na assistência ambulatorial às pessoas vivendo com HIV/Aids em Pernambuco. Ciencia \& Saude Coletiva,2012 v. 17, p. 147-156. 
6. BOTTI SHDO. Desenvolvendo as Competências Profissionais dos Residentes. Rev do Hospital Universitário Pedro Ernesto, UERJ, 2012 v. 11, p. 102-105.

7. BRANDÃO V. Caminhos do Saber: Reflexões. 1nd eed: Grupo de Estudos e Pesquisa em Interdisciplinaridade, 2015. 92p.

8. BRANDÃO VMAT. Desafios da formação interdisciplinar. 1nd ed: Kairós Gerontologia, 2009. 99p.

9. BRASIL MDE. PORTARIA INTERMINISTERIAL N 698 , DE 19 DE JULHO DE 2007. Diario Oficial da União, v. 139, n. 2, 2007.

10. BRASIL MDE. Programas de Residência em Saúde: Importância e Situação Atual. Disponível em: <http://portal.mec.gov.br/residencias-em-saude/residencia-multiprofissiona>. Acesso em: 28 jan. 2019.

11. BRASIL MDS. Comissão Nacional de Residência Multiprofissional em saúde. Diario Oficial da União, p. 24-25, 2012.

12. FAZENDA ICA. Didática e Interdisciplinaridade. 13. ed. 2008.

13. FAZENDA ICA. Interdisciplianaridade. v. 1, p. 1-105, 2014.

14. FAZENDA ICA, VARELLA AMRS, ALMEIDA TTDO. Interdisciplinaridade: Tempo, Espaços, Proposições. Revista e-Curriculum, v. 03, 2013.

15. GUEDES LE, JUNIOR, MF. Relações Disciplinares em um Centro de Ensino e Pesquisa em Práticas de Promoção da Saúde e Prevenção de Doenças. Saúde Social, 2010 v. 19, n. 2, p. 260-272.

16. HARTZ ZMDA, CONTADRIOPOULOS AP. Integralidade da atenção e integração de serviços de saúde: desafios para avaliar a implantação de um "sistema sem muros". Cad. Saúde Pública, Rio de Janeiro, 2004, v. 20, n. 2, p. 331-336.

17. IZECKSOHN MMV et al. Preceptorship in Family and Community Medicine: challenges and achievements in a Primary Health Care in progress 737. Ciência \& Saúde Coletiva, 2017 v. 22, n. 3, p. 737-746.

18. LOBATO CP. Formação dos Trabalhadores de Saúde na Residência Multiprofissional em Saúde da Família: uma cartografia da dimensão política. Dissertação (Mestrado em Saúde coletiva) UEL, 2010.

19. MITTELMARK MB et al. The Relevance of Salutogenesis to Social Issues Besides Health: The Case of Sense of Coherence and Intergroup Relations. The Handbook of Salutogenesis, 2016 p. 1-461.

20. O'REILLY P. et al. Assessing the facilitators and barriers of interdisciplinary team working in primary care using normalisation process theory: protocol for an integrative review. Plos One, 2017 v. 12, n. 7, p. 1-22.

21. REIS BAO, FARO A. A Residência Multiprofissional e a Formação do Psicólogo da Saúde: Um Relato de Experiência. Revista Psicologia e Saúde, 2016v. 8, n. 1, p. 62-70.

22. REIS MBDF. Interdisciplinaridade Na Prática Pedagógica: Um the Interdisciplinarity in Pedagogical Practice: One. Revista de Educação, Linguagem e Literatura da UEG-Inhumas, 2009 v. 1, n. 2, p. 1-7.

23. SILVA ABDA et al. Capacitação de Precptores de Enfermagem: Estratégia para Mudança na Formação em Saúde. Rev de Pesquisa: Cuido é Fundamental Online, 2010 v. 2, p. 620-622.

24. SILVA VCD, VIANA LDO, SANTOS CRGCD. Social and pedagogical practice of the nurse-preceptor: A case study, 2014 v. 13, p 102-112.

25. VASCONCELOS M, GRILLO MJC, SOARES, SM. Práticas Pedagógicas em Atenção Básica á Saúde, Tecnologias para Aboradagem ao Indivíduo, família e Comunidade. UFMG, 2009. 